\title{
Testing For Added Water in Milk with Handheld LCR Meter
}

\author{
Stojan Rendevski, Ahmed Sulaiman Ali Alghanbeer Alkhanbouli, Khaled Saif \\ Mohamed Ali Al Shaabi, Saif Abdullah Ali Ahmed \\ Faculty of Engineering and Technology, Higher Colleges of Technology, Ras al Khaimah Men's Campus, \\ United Arab Emirates
}

\begin{abstract}
Milk adulteration is a problem for the dairy industry. It can cause economic losses, lowering the quality of products and putting the consumers' safety in risk, especially milk products for children and infants. Adding water to milk is a common practice by farmers in many countries. A method of electrical conductance measurement at high frequency can give information if the milk is with added water before processing it. The objective of the research is to develop a measuring method with handheld device that can detect added water in fresh milk by measuring the electrical conductance. Namely, if there is no added water, the conductance will drop down of the initial value if we add $1 \%$ water, but will rise to initial value if we add $2 \%$ and after will decrease continuously with addition of $3 \%$ or more. If there was already added water, the conductance of the milk will decrease only with addition of water without noticeable drop of the value when we add 1-2\%.
\end{abstract}

Keywords: milk adulteration, added water, testing device, electrical conductance, LCR meter

\section{Introduction}

Milk has a great nutrition value for the humans, especially young children. Research from UNESCO and WFO showed that $80 \%$ of the food taken by six months old babies is consisted of milk and $50 \%$ of the food taken of 6 years old children is consisted of milk. The nutrition value of milk comes from its composition. The main components of milk are: proteins, lipids, lactose, vitamins, minerals and enzymes. $87 \%$ of the weight of one liter of milk goes to water, and the other $13 \%$ goes to all the other constituents. The average composition of cow milk and human milk (excluding water) is given in Table 1 [1]. Humans prefer cow milk because of its closed composition to the human's milk. Generally speaking, except for immunological constituents (antigens), cow milk has same nutrition value as the human milk.

Table 1. Composition of cow and human milk.

\begin{tabular}{|l|l|l|l|l|}
\hline \multirow{2}{*}{ Constituent } & \multicolumn{2}{|l|}{ Concentration $(\mathrm{g} / \mathrm{L})$} & \multicolumn{2}{l|}{ Proportion $(\%$ solid) } \\
\cline { 2 - 5 } & cow & human & cow & human \\
\hline Fat & 37.0 & 38 & 28.9 & 29.0 \\
\hline Protein: casein & 27.6 & 20.6 & 21.6 & 16 \\
\cline { 5 - 6 } Protein: whey & 6.4 & & 5.0 & \\
\hline Lactose & 48 & 70 & 37.5 & 53.1 \\
\hline Non-protein nitrogen & 1.9 & 0.35 & 1.5 & 0.3 \\
\hline Ash: minerals & 7.0 & 2.1 & 5.5 & 1.6 \\
\hline Total solids & 127.9 & 124.0 & 100 & 100 \\
\hline
\end{tabular}

Milk is a complex mixture of water, fat, proteins - predominantly casein, lactose, minerals and vitamins, distributed throughout the colloidal and soluble phase, explained below. In order to understand the electrical properties of milk and milk's electrical conductivity, we must determine the contribution of each separate constituent in the milk at same concentration as present in milk. The data related to the milk conductivity are given in Table 2 [2]. The data corresponds for measurement in AC electric field at $100 \mathrm{kHz}$ frequency and temperature $8^{\circ} \mathrm{C}$. It is obvious from Table 2 that the electrical conductance of milk is determined by the charged constituents, such as the minerals. Lactose does not contribute to the electrical conductance, because the conductance of full-fat milk, skimmed milk and reduced lactose milk are same. The minerals in milk are in salt form mainly of chlorides, phosphates, citrates, carbonates, potassium, sodium, calcium and magnesium. The salt concentration in milk in many cases remains unchanged at about $0.7 \% \mathrm{w} / \mathrm{v}$, the relative concentration of various ions can vary influenced from the animal breed, season of the year, feed and stage of lactation. These factors determine the concentration distribution of calcium, phosphate and magnesium ions between the soluble (water phase) and insoluble (colloid phased) of milk and thus the concentration of the free ions that contributes to the electrical conductance of milk $[2,3]$. 
Table 2. Conductance of milk and its components [1]. (Source: Henningsson, Östergren and Dejmek, 2005).

\begin{tabular}{|l|l|l|l|}
\hline Sample & Fat $(\% \mathrm{w} / \mathrm{v})$ & Lactose $($ in $\% \mathrm{w} / \mathrm{v})$ & Conductance $(\mathrm{mS})$ \\
\hline Full-fat milk & 3.6 & 4.9 & $5.05 \pm 0.03$ \\
\hline Skimmed milk & 0.1 & 4.9 & $5.40 \pm 0.03$ \\
\hline Semi-skimmed milk & 1.6 & 4.9 & $5.23 \pm 0.03$ \\
\hline Untreated milk & 3.6 & 5.2 & $4.85 \pm 0.03$ \\
\hline Reduced lactose milk & 3.6 & 0 & $5.00 \pm 0.03$ \\
\hline NaCl $(1 \mathrm{~g} / \mathrm{L})$ & & & $2.50 \pm 0.03$ \\
\hline KCL $(1 \mathrm{~g} / \mathrm{L})$ & & & $2.26 \pm 0.03$ \\
\hline Sodium caseinate $(3.5 \% \mathrm{w} / \mathrm{v})$ & & & $0.10 \pm 0.03$ \\
\hline Ultrapure water & & & $<.001$ \\
\hline
\end{tabular}

Casein, the main protein in the milk, gives very small conductance compared to the mineral salts. However, it can still influence milk conductance. Casein proteins mostly exist in the colloidal phase. Calcium phosphate salts can be found in the casein micelles. A small part of the sodium and potassium ions are linked to the casein as counter-ions to the negatively charges organic phosphate groups in the protein. These mineral ions act like bridges between the subunits of the casein micelles and keep the milk in a stable condition. The salts can be released into solution thereby increasing the conductivity. It has been found in the literature that the conductance of full fat milk increases by approximately $15 \%$ when the milk is left at room temperature for $48 \mathrm{~h}$. It should be noted that the change in conductance between full fat and skimmed milk is only 5-10\% (Table 2). This suggests that the changes in the conductivity observed as the milk goes-off is not due solely to the deformation of the fat globules. Over time, the links between the subunits in the casein micelles break down and release free ions, mainly calcium. As a consequence, the milk conductance increases.

Electrical conductance of milk increase with acidification. In one experiment described in the literature, when freshly squeezed lemon juice was added to the full fat milk, the conductance increased, reaching saturation value of $5.8 \mathrm{mS}$ at $\mathrm{pH}=5.0$ [7]. This behavior is due to the fact that all the colloidal mineral salts connected to the micelles are in a soluble phase now and are free to contribute to the measured electrical conductance.

Fat has an influence on the milk's conductivity, also. Electrical conductivity of milk decreases with increase of percentage of fat. The explanation on the cause of the decrease in conductance is that $97 \%$ of the total milk fat is in the large globules enveloped by thin nonconductive membrane. The globules hinder the electrical conductance by occupying the volume of the conducting medium and opposing the mobility of the free ions. Most of the fat globules are with size in wide range $(2-10 \mu \mathrm{m}$ in diameter $)$, depending on the breed and the season of the year, influencing the conductance accordingly. Larger fat globules give smaller contribution to the milk electrical conductance [4]. The conductance of fresh full-fat milk compared to the untreated milk with the same fat concentration (Table 2) is attributed to the reduction in size of the fat globules by the treatment (pasteurization and homogenization). The electrical conductance of mill left at room temperature for 48 hours increases followed by saturation. This behavior can be attributed to the clumping of fat globules resulting from the disruption of the thin fat globule membrane, releasing free fatty acids. Approximately $50 \%$ of the phospholipids occur in the globule membrane and are released into the milk solution producing free phosphate ions. At the same time, the acidity of the milk starts to increase releasing calcium ions to contribute to the conductivity. For the skimmed milk, the effect of the fat globules is very limited as the percentage of fat is less than $0.1 \%$. Therefore, the conductance of skimmed milk shows smaller increase than the conductance of full fat milk.

Beside mineral salts and fat globules, lactose and proteins are the other main constituents of milk. Lactose molecules do not have a charge, and lactose can affect electrical conductivity only by its effect on milk viscosity. Proteins do have a net charge depending on the $\mathrm{pH}$ and their mobility is directly measured by electrophoresis. Proteins affect the conductivity in two different ways: by having a charge and by influencing the viscosity. Proteins in milk can be considered as macro ions, while minerals can be considered as micro ions. The electrical conductivity contribution of the macro ions to the total milk conductivity can be found by knowing the net charge and the size [3]. If we take beta-lactoglobulin as a dominant whey protein in the milk, which has a charge of about $7 \mathrm{e}$ (the unit charge of the electron or the proton, $\mathrm{e}=1.6 \cdot 10^{-19} \mathrm{C}$ ) at physiological $\mathrm{pH}$, and radius of $2.5 \mathrm{~nm}$, the ionic molar conductivity (of 1 mole concentration of beta-lactoglobulin) is equal to:

$$
\Lambda=\frac{F e z^{2}}{6 \pi \eta r}=80 \mathrm{~S} \cdot \mathrm{cm}^{2} / \mathrm{mol}
$$

where $F$ - Faraday's constant, e - elementary charge, $\mathrm{z}$ - valence of the macro ion, $\eta$ - viscosity. If the concentration of the beta-lactoglobulin in milk is $0.3 \% \mathrm{w} / \mathrm{v}$; which is equal to $0.2 \mathrm{mM}$, then the contribution to total milk electrical conductivity would be less than $0.5 \%$. 
Electrical conductivity of milk is lower than for its fat and casein free phase due to obstruction of mobility of the charge carrying free ions. The relation between the fat concentration and specific electrical conductivity of milk is very well investigated and studied experimentally, and is given by the formula:

$$
\sigma=\sigma_{0} \cdot v^{3 / 2},
$$

where, $\sigma_{0}$ - specific conductivity of the fat-free milk and $v$ - volume fraction of the conducting medium. The difference of specific electrical conductivity of fat-free milk and full-fat milk is $0.27 \%$ for milk with fat concentration of $4 \% \mathrm{w} / \mathrm{v}$.

The ionic conductivity of milk is mainly due to the presence of $\mathrm{Na}^{+}, \mathrm{K}^{+}$and $\mathrm{Cl}^{-}$ions. However, the variation in the fat globule size and differences in the casein protein structure contributes to the total electrical conductivity of the milk. In the aspect of the project activities taken which involved steering the milk and adding water (changing concentration of the main milk constituents), it is to be expected that at some critical concentration of added water, the milk conductivity can change the value in a way that is predictable (in correlation) to the changes in the milk concentration. That has been the main objective of the project.

Electrical conductivity method is one of the testing method used for determination of quantities such as soluble mineral salts, protein content in whey powder, casein content during renneting (cheese making), control of the pasteurization, detection of intra-mammary infection (mastitis) and sanitary cleaning as a quality control indicator [5].

Electrical conductivity of milk increases by increasing the temperature, mainly caused by decrease of the viscosity. Other effects can influence conductivity, such as changes in the degree of dissociation of the mineral salts and changes in the hydrodynamic radius of the movable charges particles. Dissolution of calcium and phosphate from the casein micelles and formation of calcium phosphate $\mathrm{Ca}^{3}\left(\mathrm{PO}_{4}\right)_{2}$ is the main contributor to conductivity change here. When $\mathrm{pH}$ decreases, protons are released in milk (positive hydrogen ions), but below $60{ }^{\circ} \mathrm{C}$, the changes on milk conductivity are negligible. The temperature coefficient of milk conductivity in interval $15-40{ }^{\circ} \mathrm{C}$ has been found small, or $1.70 \cdot 10^{-3} \mathrm{mS} /{ }^{\circ} \mathrm{C}[3]$.

In case of cream, sour cream or yogurt fermentation that lead to milk acidification, the time of termination of the fermentation is a crucial point in production of dairy products. Mixed lactic acid bacteria are used as starters of the fermentation processes. These bacteria acidify the milk by formation of lactic acid from lactose present in the milk. Consequently, evolution of milk acidification is important and is generally followed by measuring $\mathrm{pH}$ with glass electrodes. Many governmental legislations do not allow use of glass electrodes during the production of fermented products, aimed to prevent shattering of the glass $\mathrm{pH}$ electrodes and therefore, possible damage to the consumers. In the routine work in the dairy industry, samples must be taken periodically for analysis, which is labor intensive work. Electrical conductivity of fermented milk is determined by lactic acid production and by the concentration of solubilized anions and cations [6]. The method of electrical conductometry with metal or conductive polymer electrodes is a suitable replacement for this purpose.

Intra-mammary infection, such as mastitis, causes increase in electrical conductivity of milk. Cells at the mammary epithelial cells are damaged during inflammation, resulting in increase of free ions from the cells in the lactating lumen and thus increase of the electrical conductivity of milk [7].

Milk adulteration makes significant problems for the dairy industry. It leads to economic losses, deterioration of the quality of the products, and is a risk for consumers' safety. Adding water to milk is a common practice by farmers in many countries. Dairy industry is not much in care, because they pay the farmers by unit of fat or unit of protein they test before milk is taken in production. If a farmer adds $25 \%$ water to milk, concentration of the fat and protein drops down for $25 \%$. What farmers get in volume they lose on price. It is a different story if the milk is to be taken from the farmers for special purposes, for example, baby food production, short shelf-life dairy products. Water from the farmers can be contaminated with pathogenic bacteria or can have salinity that is over the allowed standard upper limits. In such cases, dairy industry is obliged to check if there is added water in the milk, or the milk is untreated and fresh. Another reason why dairy industry wants to know if farmers add water is to follow seasonal and regional changes in milk supply. If farmers add water to milk, that means they produce less milk then agreed and reasons must be known by the dairy industry. There can be long-lasting negative effects on the steady development of the industry if there is no way to control farmers if they add water in order to deliver agreed quantity.

In practice, there are techniques based on the changes in freezing point of the milk (cryoscopy method) or on changes in the refraction of light of the whey component of milk after the removal of the fat components, with which added water can be tested. These techniques are expensive and time consuming. Other methods that are fast, cheap and reliable are requested.

Recently, a new cheap and fast method has been developed for testing added water in milk based on interdigital capacitance sensor [8]. It has been found that by increasing the amount of water from $10 \%$ to $50 \%$ at the frequency $1 \mathrm{kHz}$, the value of electrical capacity of fresh milk rose from $17.09 \mu \mathrm{F}$ to $20.20 \mu \mathrm{F}$ and the value 
of electrical capacitance $C$ increased from $7.69 \mu \mathrm{F}$ to $8.66 \mu \mathrm{F}$ at the frequency of $10 \mathrm{KHz}$. When the measurement of electric power is at a frequency higher than $1 \mathrm{kHz}$; it has been found that the value of electrical capacitance decrease respectively. In this study, addition of less than $10 \%$ water to milk were not studied. However, the greatest disadvantage of the method of capacitance sensor lays in the fact that milk samples with different lactose concentration can have the same capacitance as fresh milk with different protein concentration, or somatic cells count. In order to have reliable data for added water in milk with the capacitance sensor method, one must know exact number of somatic cells, lactose and proteins concentration $[9,10]$. This make the method not suitable for direct application on farms when collecting the milk by the dairy industry. Collectors from the dairy industry must use faster and more reliable method for testing added water in fresh milk. We developed such a method in this project and have proved to be fast, reliable and cheap - the method of $100 \mathrm{kHz}$ conductance of platinum electrodes immersed in milk. The handheld device we developed is cheap (costs less than 250 USD), reliability has been proven to be $\pm 0.05 \mathrm{mS} / \mathrm{cm}$ on conductivity and the time for getting data on added water is less than 30 seconds.

Electrical conductance $G$, the reciprocal quantity of the resistance $R(G=1 / R)$, is measured in basic unit of Siemens (S). The conductance $\mathrm{G}$ is dependent on specimen dimensions (distance between the electrodes and area of the electrodes. When conductance is expressed without dimensions, it's called electrical specific conductivity $\sigma$ (in units of $\mathrm{S} / \mathrm{m}$ or $\mathrm{S} / \mathrm{cm}$ ). It's related to the specific resistance $\rho$ as $\sigma=1 / \rho$ :

$$
R=\rho \frac{L}{A} ; \quad G=\frac{1}{R}=\frac{1}{\rho} \frac{A}{L}=\sigma \frac{A}{L} .
$$

The DC conductance of the milk as an electrolyte is simply measured by immersing two electrodes into the solution (milk) and applying a DC voltage. According to the Ohm's law, Conductance is the ratio of the current to the voltage measured.

Measurements involving DC voltage may lead to electrolysis of water in the solution and electrode polarization, which reduce passed current through the circuit to zero in short time. This unwanted situation in practice is eliminated by using $\mathrm{AC}$ voltage. When $\mathrm{AC}$ voltage is applied to the sample, the in-phase current (related to the conductance via the resistance and the Ohm's law) and the out-of-phase current (related to the capacitance $C$ are measured over a range of frequencies using an impedance analyzer or LCR meter. The method is called admittance spectroscopy.

The measured conductance $G$ and susceptance $B$ of a salt solution always show the effect of electrode polarization (Fig.1) [11]. On figure 1, the low frequency region $(<1 \mathrm{kHz})$ is related to the electrical characteristics of the interface between the electrodes and the solution (milk). The high frequency region $(>1$ $\mathrm{kHz}$ ) is in connection to the electrical conductance of the bulk solution (milk only, excluding the electrodes polarization). The frequency at which the susceptance reaches maximum is called relaxation frequency of the electrode polarization.

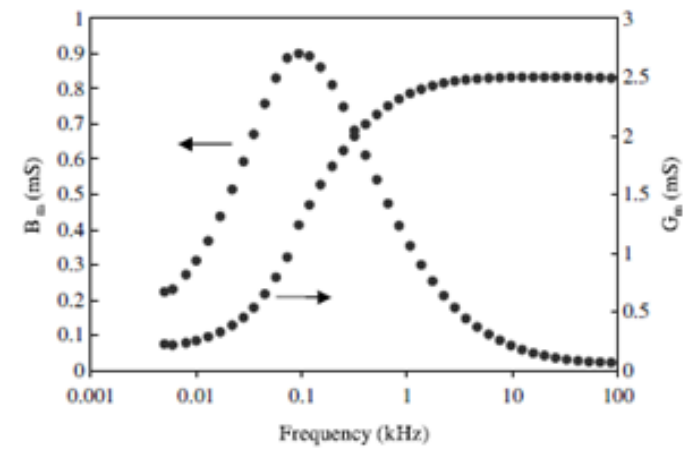

Fig.1. Conductance $G$ and susceptance $B$ of $1 \mathrm{~g} / \mathrm{L} \mathrm{NaCl}$ in water. Source: Mabrook and Petty (2003).

The impedance analyzer method (admittance spectroscopy) is an expensive method. The instruments, the electrodes with cables, cost around 10,000 USD. We are on an opinion that simple LCR meter that costs below 250 USD and simple pH electrodes made from platinum wires, not plates, that costs bellow 100 USD, can serve for the purpose of testing for added water in fresh milk. 


\section{Experimental methods and results}

Main material which was used for research in the study is milk. We went in middle of February 2107, to a farmer in Shamal village in Ras al Khaimah, UAE, to collect fresh milk from a local Arabian breed cow (figure 2 and figure 3). A volume of $20 \mathrm{ml}$ has been proved in the research that is more than enough. We collected $200 \mathrm{ml}$ fresh milk.

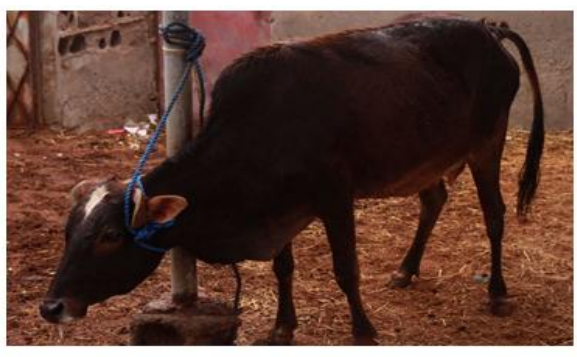

Fig.2. Local Arabian breed cow.

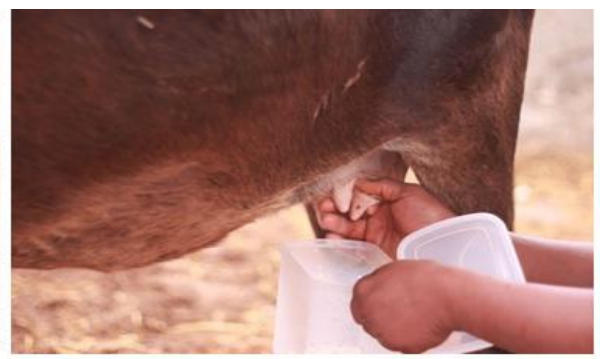

Fig.3. Fresh milk collecting.

The electrodes we used should be from non-corroding metal, such as from platinum. The platinum electrodes were important part of electrical conductance measurement. We purchased electrodes type EC505 from Extech, USA, through the local distributor company Anaum from Dubai. In order to obtain the cell constant of the electrodes, $1.00 \mathrm{~g} / \mathrm{L}$ of $\mathrm{NaCl}$ were prepared in distilled and deionized water. The specific conductivity of the sodium chloride salted water is known at $2.02 \mathrm{mS} / \mathrm{cm}$ at $25{ }^{\circ} \mathrm{C}$. The LCR instrument we used for AC conductance measurement at $100 \mathrm{kHz}$ is model type DE-6000 from IET Labs, USA, supplied through the local distributor company Anaum from Dubai. The LCR meter is a high-performance LCR meter that is full-featured and cost effective. The $0.2 \%$ basic accuracy and optional USB interface for data transfer. The DE-6000 measures: $L s / L p$-- Series and parallel inductance; $C s / C p$-- Series and parallel capacitance; $R s / R p$ -- Series and parallel resistance (ac); $R d c / R p$-- Series and parallel resistance (dc); ESR/Rp -- Series and parallel equivalent resistance; $D$-- Dissipation factor; $Q$-- Quality factor; $\Theta$-- Phase angle. The voltage test signal level is $0.5 \mathrm{Vrms}$. For the temperature measurements we used integrated circuit temperature sensor type LM335 (Texas Instruments, USA), with accuracy of $0.1{ }^{0} \mathrm{C}$. For dilution of milk with addition of water, we used distilled and deionized water supplied from the local car battery shop.

Several procedures need to be clarified here in the research method description.

1) The electrodes must be fully immersed in the milk testing sample.

2) After addition of desired quantity of water, gentle steering of the milk is needed, avoiding air bubbles creation.

3) The measurement of conductance should be taken after stabilization of the readings on the LCR meter, usually after 20 seconds.

4) Careful cleaning of the electrodes is needed after use. We worked with detergent and washing the electrodes many times with distilled water after. No single scratching has been made on the electrodes during the cleaning.

The measured data were imported in a graphical analysis software OriginPro, USA. The results are shown in the bellow section of the report. The measurement of the electrical conductance has been made for fresh milk and commercial low fat milk. First, the volume of the starting milk was known. After addition of proper volume of water, we calculated the $\%$ of added water. The measured electrical conductance taken at 100 $\mathrm{kHz} \mathrm{AC}$ voltage of $0.5 \mathrm{~V}$ between the electrodes were recorded in the graphical analysis software, OriginPro, USA. The results of the measurements for the commercial low fat milk are shown on figure 4. For the purpose of better visibility of the data at lower added water content, were we expect anomaly of the conductivity (nonlinearity), we show also the same data in the so called logarithmic plot (figure 5).

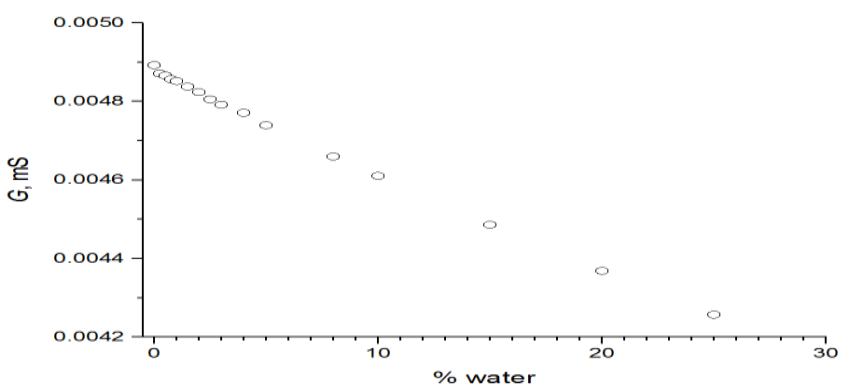

Fig.4. Normal plot of the electrical conductance of commercial low fat milk. 


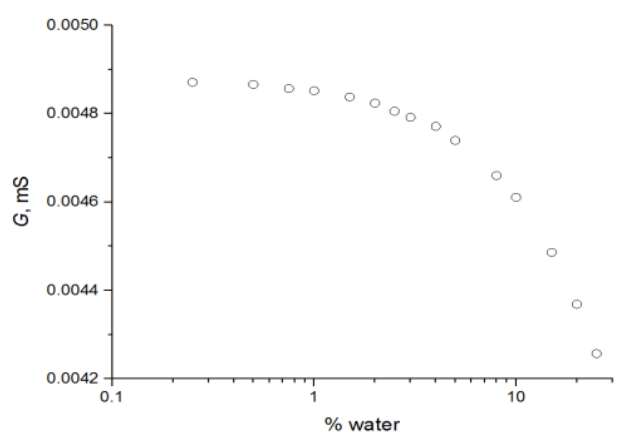

Fig.5. Logarithmic plot of the electrical conductance of commercial low fat milk.

The results of the measurements for the fresh milk supplied from the farm are shown on figure 6. For the purpose of better visibility of the data at lower added water content, were we expect anomaly of the conductivity (non-linearity), we show also the same data in the so called logarithmic plot (figure 7).

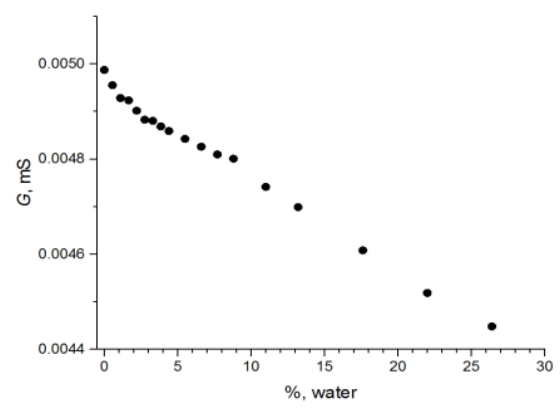

Fig.6. Normal plot of the electrical conductance of fresh milk from the farm.

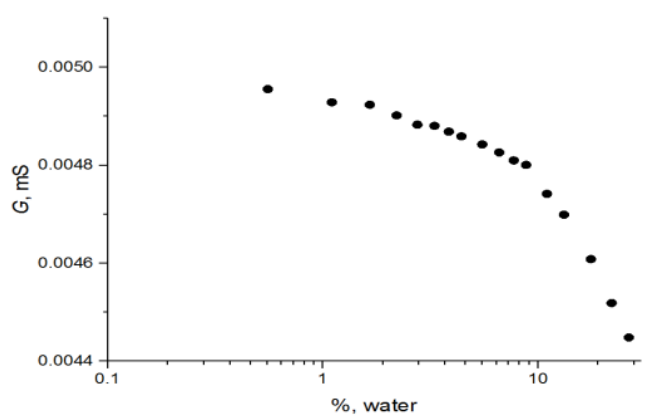

Fig.7. Logarithmic plot of the electrical conductance of fresh milk from the farm.

\section{Discussion}

The electrical conductance of the fresh milk showed anomaly at $2 \%$ added water (fig. 8 ). Namely, the conductance at $2 \%$ added water in milk is lower than at $2.5 \%$ water. This behavior has not been noticed on the commercial low fat milk sample (fig.5).

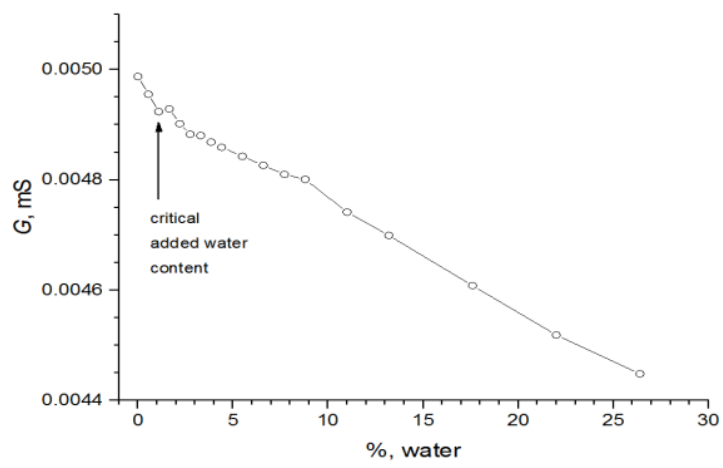

Fig.8. Detected anomaly of the electrical conductance of fresh milk at $2 \%$ added water. 
Here lies the advantage of the proposed research method. If the milk taken from the farm is without added water, after we add $2 \%$ water, this behavior will be seen. If the farmer added $2 \%$ water or more, and usually they add more than $20 \%$, this behavior of the electrical conductance cannot be seen. The important thing here is not to pass $2 \%$ added water in the milk during the testing. The testing of added water can be very simple in practice. Take the milk, add $2 \%$ water and $3 \%$ water. No need to add more water. If electrical conductance of the milk at $2 \%$ is lower than at $3 \%$ added water, the milk is fresh, If not, the milk is with added water. Simple and fast. We managed to do the testing for less than 30 seconds. It proved that the milk was without added water as we observed the same during collecting it from the farmer (figure 7).

Another peculiar behavior has been seen on the logarithmic plot of the same data from fig. 8 for the fresh milk without added water prior the testing. This behavior is shown on figure 9 .

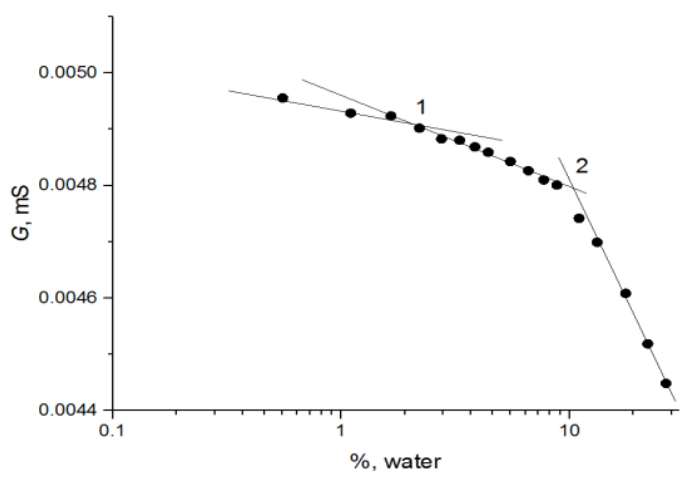

Fig.9. Peculiar behavior of electrical conductance of fresh milk at two different added water content.

The dependence of the electrical conductance on figure 9, showed three linear parts, instead of two. At the boundary of the first two, at concentration around $2 \%$ added water, we observed a break-point, which was expected. We already observed anomaly in the electrical conductance at $2 \%$ added water (figure 8 ). However, we observed another anomaly in the electrical conductance behavior at added $10 \%$ water. This is the second break-point in the electrical conductivity. In order to make firm conclusion on the cause of this peculiar behavior, we think more research is needed in future on the hygienic condition of the fresh milk.

\section{Conclusion}

It is known from practice that milk adulteration makes significant problems for the dairy industry. It leads to economic losses, deterioration of the quality of the products, and is a risk for consumers' safety. The effects on the dairy industry can be negative for the steady development of the industry. The control of quality of delivered milk is an important practice in the chain of total quality management in the dairy industry.

Techniques based on the changes in freezing point of the milk or changes in the refraction of light of the protein component of milk after the removal of the fat components, are expensive and time consuming. These techniques are expensive and time consuming. Our proposed method of conductance testing with a handheld device is much cheaper and very fast. We have proved this with great satisfaction.

A method to detect added water to full fat untreated milk has been developed using electrical conductance measurements with AC voltage of $0.5 \mathrm{~V}$ between platinum electrodes at frequency of $100 \mathrm{kHz}$. The characteristics of electrical conductance of skimmed milk from the industry at $100 \mathrm{kHz}$ and $25{ }^{\circ} \mathrm{C}$ showed a linear decrease in conductance with increasing water content over a wide range of water concentrations from 0 to $25 \%$. On the other hand, the conductance of fresh and untreated milk showed a decrease only if added water concentrations is higher than $2 \%$. At lower added water concentrations, between 0 and $2 \%$, fresh milk showed anomalous conductance minimum at around $1.5 \%$ and reached maximum again, when the added water to the milk was found around 2-2.5\%.

The work shows that milk conductance $G$ at $100 \mathrm{kHz}$ decreases approximately linearly as the concentration of water in the milk increases. These results indicated that the high frequency saturation value is a property of the bulk milk solution. In contrast, the low frequency region represents the properties of the interface between the electrodes and the milk solution, and we avoided that with $100 \mathrm{kHz}$ AC voltage between the platinum electrodes. The increase in milk conductance at $2 \%$ added water can be explained by the hydrolysis of milk fat to its subunits. Hydrolysis is a chemical reaction by which chemical bonds are broken by the insertion of water between the atoms involved in the bond. The salts present in milk in the colloidal phase usually catalyze this reaction. As milk fat is composed of three fatty acids covalently bound to a glycerol molecule by ester bonds, these bonds may hydrolyze on adding around $2 \%$ water, producing sodium and calcium salts of fatty acids. This increases the number of conducting ions in the milk solution and, consequently, increases the 
conductance. Approximately $50 \%$ of the phospholipids that occur in the fat globules' membranes are released into the milk solution due to the hydrolysis process producing free phosphate ions $[7,11,12]$. That is another source of free ions which increases the milk conductance.

From a practical point of view, we found that the conductance of fresh milk with $2 \%$ added water is higher than the conductance at $1 \%$ added water. That means the milk is good and there is no added water. However, when the conductance of fresh milk with $2 \%$ added water is lower than the electrical conductance of milk with $1 \%$ added water - that means there is at least $1 \%$ added water or more to the original sample before testing it. In our case, the limit of detection is $\pm 0.5 \%$ water, depending on the accuracy range of conductance measurements. We worked with LCR meter with conductance accuracy of $\pm 0.01 \mathrm{mS}$. The higher electrical conductance of the fresh milk compared with the treated milk with same fat content is due to the reduction in size of the fat globules to less than $2 \mathrm{~nm}$ during milk processing.

\section{Acknowledgements}

Literature review we have made was of crucial importance for the project in almost every phase of the research, starting from the idea elaboration, justification of the project, hypothesis and objectives setting and data analysis. We acknowledge Higher Colleges of Technology for having great library up-to-date resources. The authors of the project acknowledge the help given from Mohamed Ahmed Hasan Rabeea Al Shehhi, librarian at the Ras al Khaimah Men's Campus, Higher Colleges of Technology, on leading us through the rich library resources we searched for days and days. We acknowledge Anas Abdulkadar, technician at HCT, Ras al Khaimah Campuses, for helping us finding appropriate temperature probes.

References

[1]. M. Henningsson, K. Östergren, P. Dejmek, The electrical conductivity of milk - The effect of dilution and temperature, International Journal of Food Properties, 8(1), 2005, 15-22.

[2]. L.O. Figura, A.A Teixeira, Food Physics, Physical Properties - Measurement and Applications (Berlin-Heidelberg, Springer, 2007).

[3]. M. Kent, Electrical and Dielectrical Properties of Food Materials (London, Science and Technology Publishers, 1987).

[4]. B.A. Lawton, R. Pethig, Determining the fat content of milk and cream using AC conductivity measurements, Measurement Science and Technology. 4, 1993, 38-41.

[5]. E. Norberg, H. Hogeveen, I.R. Korsgaard, N.C. Friggens, K.H. Sloth, P. Løvendahl. Electrical conductivity of milk: ability to predict mastitis status. Journal of Dairy Science, 87(4), 2004, 1099-107.

[6]. A.R. Saeed Al Hilphy, Electric field (AC) for non-thermal milk pasteurization, Journal of Nutrition and Food Science, 2, $2012,177$.

[7]. G. Mucchetti, M. Gatti, E. Neviani, Electrical conductivity changes in milk caused by acidification: determining factors, Journal of Dairy Science, 77, 1994, 940-944.

[8]. Phimphisan, W. Sangiamvibool, Determination of Water added in Raw Milk using Interdigital Capacitor Sensor, Przeglad Elektrotechniczny ISSN 0033-2097, R. 91 NR 9/2015.

[9]. S.C. Mukhopadhyay, C.P. Gooneratne, G.S. Gupta, S.N. Demidenko, A low-cost sensing system for quality monitoring of dairy products. IEEE Transactions on Instrumentation and Measurement, 55(4), 2006, 1331-1338.

[10]. N. Angkawisittpan, T. Manasri . Determination of Sugar Content in Sugar Solutions using Interdigital Capacitor Sensor, Journal of the Institute of Measurement Science, 12, 2012, 8-13.

[11]. M.F. Mabrook, M.C. Petty, Effect of composition on the electrical conductance of mil. Journal of Food Engineering, 60, 2003, 321-325.

[12]. R. Żywica, J. Budny, Changes of selected physical and chemical parameters of raw milk during storage, Czech Journal of Food Sciences, 18(245), 2000, 241-242. 